\title{
Laser-induced degradation and damage morphology in polymer optical fibers
}

Kiedrowski, K., Jakobs, F., Kielhorn, J., Johannes, H.-H., Kowalsky, W., et al.

K. Kiedrowski, F. Jakobs, J. Kielhorn, H.-H. Johannes, W. Kowalsky, D. Kracht, I. Balasa, D. Ristau, "Laser-induced degradation and damage morphology in polymer optical fibers," Proc. SPIE 11355, Micro-Structured and Specialty Optical Fibres VI, 1135504 (1 April 2020); doi:

$10.1117 / 12.2553999$

SPIE. Event: SPIE Photonics Europe, 2020, Online Only 


\title{
Laser-induced degradation and damage morphology in polymer optical fibers
}

\author{
K. Kiedrowski*a, F. Jakobs ${ }^{\mathrm{b}}$, J. Kielhorn ${ }^{\mathrm{b}}$, H.-H. Johannes ${ }^{\mathrm{b}, \mathrm{c}, \mathrm{d}}$, W. Kowalsky ${ }^{\mathrm{b}, \mathrm{c}, \mathrm{d}}$, D. Kracht ${ }^{\mathrm{d}, \mathrm{e}}$, I. \\ Balasa $^{\mathrm{e}}$, D. Ristau ${ }^{\mathrm{a}, \mathrm{c}, \mathrm{d}, \mathrm{e}}$ \\ aInstitut für Quantenoptik, Leibniz Universität Hannover, Welfengarten 1, 30167 Hannover, \\ Germany; 'bnstitut für Hochfrequenztechnik, TU Braunschweig, Schleinitzstraße 22, 38106 \\ Braunschweig, Germany; ${ }^{\mathrm{C}}$ Academic Alliance Braunschweig - Hannover Quanomet, Germany; \\ ${ }^{\mathrm{d}}$ Cluster of Excellence PhoenixD, Hannover, Germany; ${ }^{\mathrm{e}}$ Laser Zentrum Hannover e.V., \\ Hollerithallee 8, 30419 Hannover, Germany
}

\begin{abstract}
The radiation of pulsed laser systems can generate changes in various materials. On the one hand, these modifications can be used for a variety of applications i.e. laser welding, cutting and many more [1]. The precision and quality depends on the material and laser parameters. On the other hand, material changes are not always desired in other applications. When using optical materials such as optical fibers as a light guide or as a sensor, laser-induced damage effects inside the fiber are to be prevented to ensure constant light guidance and the reliable monitoring of a desired parameter. Therefore, investigations for quality assurance need to be performed. For this reason, this work investigates laserinduced damage in polymer optical fibers (POF) using a nanosecond pulsed laser system at a wavelength of $532 \mathrm{~nm}$. The impact of different laser and fiber parameters on the long-term degradation behavior is observed. In addition, the overall degradation behavior as well as the knowledge gained by analyzing the damage morphology and distribution will be used to obtain a better understanding of the damage mechanisms.
\end{abstract}

Keywords: polymer optical fiber, active POF, laser damage, PMMA, integrated photonics

\section{INTRODUCTION}

Polymer optical fibers (POF) have many applications, but the number of investigations on their optical radiation resistivity is relatively low. As a result, the optical radiation resistivity of the fiber preform material is often used as a reference value. The problem with this approach is that the fiber drawing process can create a larger number of imperfections and introduce further contaminations in the optical material. Moreover, the drawing process itself causes changes in the material stress. All these modifications of the pristine material can lead to a lower laser-induced damage threshold (LIDT) of polymer optical fibers compared to the preform material. Gebauer et al. [2] investigated the LIDT of silica fibers and found a fluence of approximately a factor 23 lower than that of the pure silica material. Therefore, it is also of high importance to increase the knowledge concerning the laser-induced damage in POFs and to study the causes behind the damage mechanisms.

In the UV regime Wandschneider et al. [3] investigated the transmission properties of PMMA based POFs propagating pulsed radiation at $337 \mathrm{~nm}$ and $355 \mathrm{~nm}$, respectively. However, in the present work a pulsed Nd:YAG laser irradiating at a wavelength of $532 \mathrm{~nm}$ and a pulse duration of $4.4 \mathrm{~ns}$ is used to induce damages in POFs with PMMA as the core material. Previous measurements [4] focused on the short-term degradation applying R-on-1 tests in which a number of 1,000 pulses were coupled into the fiber. Afterwards, the pulse energy was increased, and the same number of pulses were propagated through the fiber. This procedure was repeated, and small damages were observed during this approach. However, the measurement was stopped when a specific catastrophic damage was monitored. This first study showed an LIDT of $0.32 \mathrm{~J} / \mathrm{cm}^{2}$ for the catastrophic damage of the POFs compared to an LIDT of $0.57 \mathrm{~J} / \mathrm{cm}^{2}$ of the preform. The preform LIDT is in good agreement with measurements done by Melninkaitis et al. [5] on a stretched PMMA waveplate who determined a value of $0.46 \mathrm{~J} / \mathrm{cm}^{2}$ using a laser system with slightly different parameters. The first visible structural change in the POF before catastrophic damage occurs at even lower fluence of approximately a factor of 20.

*kiedrowski@iqo.uni-hannover.de; phone +49 5112788 363; fax +49 5112788100 
Complementary the previous investigations with a rather small number of pulses, this study focuses on the long-term degradation of POFs by coupling at least 40,000 pulses into the fiber sample. The influence of different pulse energies, the polymer chain length and in case of active fibers, the concentration of Rhodamine B on the fiber degradation was investigated. Rhodamine B was chosen because it is a common laser dye for active polymer optical fibers absorbing at a wavelength of $532 \mathrm{~nm}$. The monitored fiber transmission, the relative scatter loss along the fiber, as well as the damage distribution and morphology are used to characterize the fiber degradation.

\section{FIBER PREPARATION AND EXPERIMENTAL SETUP}

\subsection{Preparation of the polymer optical fibers}

Our project partner, the TU Braunschweig, manufactured the polymer optical fibers used in this investigation, and the corresponding fiber properties are listed in table 1 . The fibers were first cut into $20 \mathrm{~cm}$ long pieces. Afterwards, the fiber facets were polished by hand using polishing sheets followed by removing remaining contaminations using air pressure. The result of each preparation step and the final result of one polished and cleaned fiber facet is shown in figure 1.

Table 1. Properties of the POFs used in this work.

\begin{tabular}{|c|c|}
\hline \multicolumn{2}{|c|}{ Fiber properties } \\
\hline $\begin{array}{l}\text { Material core/cladding } \\
\text { Diameter core/fiber } \\
\text { Refractive index core/cladding @852 nm } \\
\text { Attenuation@ } 532 \mathrm{~nm}\end{array}$ & $\begin{array}{l}\text { PMMA/404F acrylate } \\
\text { approx. } 450 / 520 \mu \mathrm{m} \\
1.49 / 1.40 \\
\text { approx. } 2 \mathrm{~dB} / \mathrm{km}\end{array}$ \\
\hline
\end{tabular}
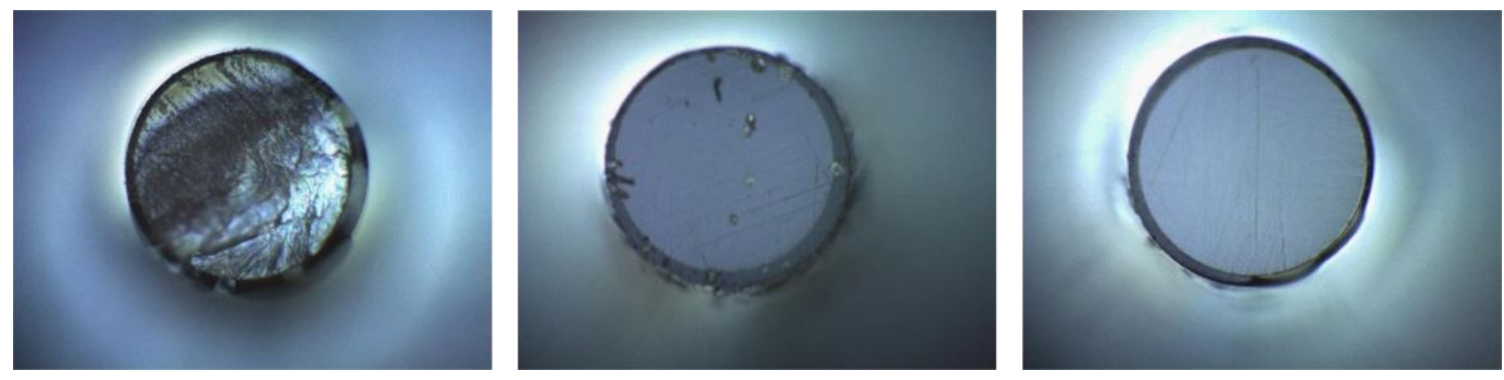

Figure 1. A polished and cleaned fiber facet after cutting (left), polished surface (center), and cleaned after polishing (right).

\subsection{Experimental setup}

An overview of the experimental setup is shown in figure 2 (left). A variable attenuator is used to vary the pulse energy emitted by a Nd:YAG laser with frequency doubling to a wavelength of $532 \mathrm{~nm}$, a pulse duration of $4.4 \mathrm{~ns}$ and a repetition rate of $10 \mathrm{~Hz}$. The laser pulses are coupled into the POF using a lens with a focal length of $250 \mathrm{~mm}$. The fiber is placed behind the focus of the lens at which the beam illuminates approximately the whole fiber facet. The corresponding beam profile is shown in figure 2 (right). Afterwards, the fiber position is adjusted until the transmission efficiency of the fiber is maximized whereby the distance to the focus is not changed. This procedure is done using the pyroelectric energy sensor ES111C by measuring the pulse energy between lens and fiber and the fiber output energy. The mean value of the transmission efficiency was approximately $83 \%$ and is in good accordance with previously measured and expected efficiencies in consideration of optical losses such as fiber attenuation and Fresnel losses. The pyroelectric energy sensor is also used to measure the pulse energy between lens and fiber for calibration of the reference photodiode voltage (PD-Ref). Hereinafter, pulse energy values are related to the pulse energy between lens and optical fiber determined by the monitored voltage of the calibrated reference photodiode. 
Laser-induced damage is well known to increase scattering and absorption. Therefore, scattered light along the fiber is monitored with a scanning photodiode and a CCD camera giving information of the damage growth. Moreover, the fiber transmission is monitored by oberserving the ratio of the transmission photodiode (PD-T) and the reference signal (PDRef) to determine the effect of the laser-induced degradation on the fiber output energy.
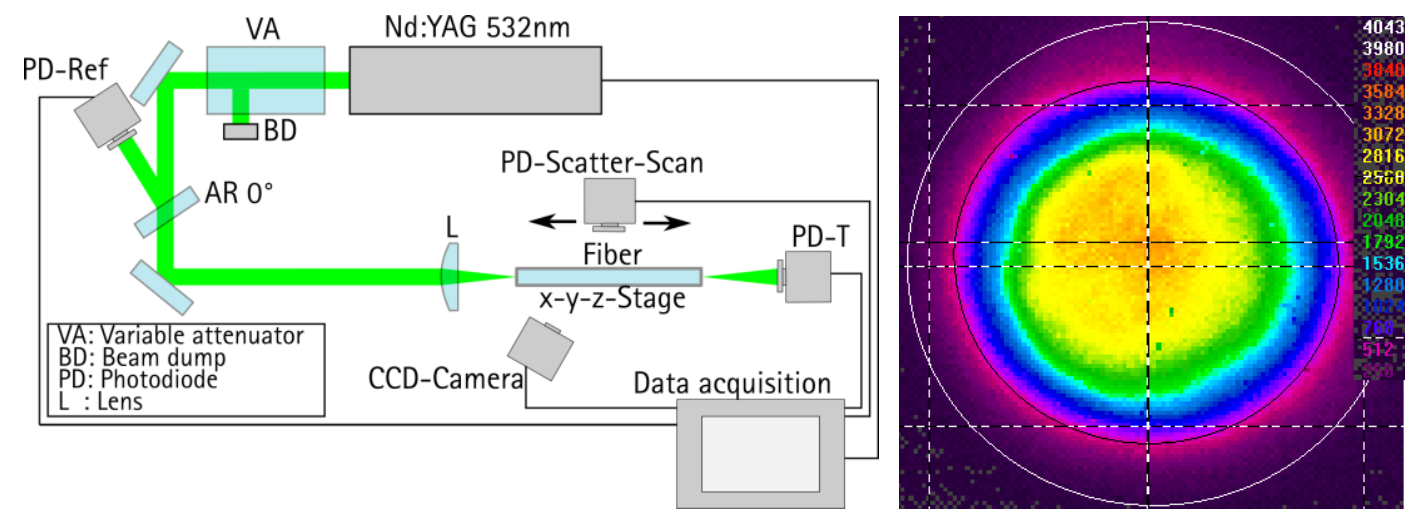

Figure 2. The measurement setup used to investigate the laser-induced degradation of optical fibers (left). The beam profile of the laser close to the fiber front facet (right).

\section{DEGRADATION OF POLYMER OPTICAL FIBERS}

\subsection{S-on-1 measurement routine}

This study focuses on the long-term behavior of the fiber degradation. For this reason, S-on-1 tests were performed. In this case, a number of at least 40,000 pulses with a fixed pulse energy were coupled into the fiber, and the relative transmission over time was monitored. For passive POFs the influence of the pulse energy as well as the polymer chain length was investigated. Furthermore, the impact of the Rhodamine B concentration on the fiber degradation is observed regarding active POFs.

A typical trend of the fiber degradation caused by the propagated laser pulses is shown in figure 3.

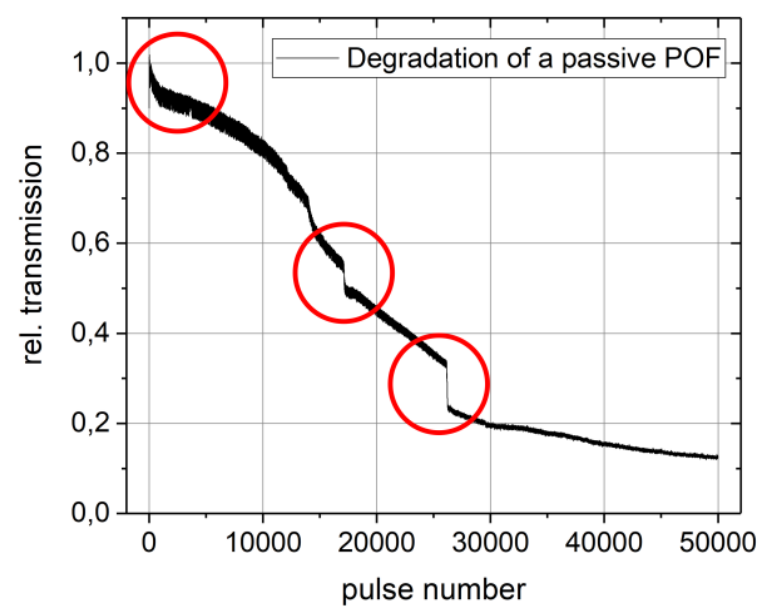

Figure 3. The relative transmission signal detected by the two photodiodes PD-T and PD-Ref as a function of the propagated pulses. 
The overall relative transmission shows an "S"-shaped curve throughout the exposure time with some significant rapid transmission losses within several hundred pulses (red circle). For each of the mentioned parameter studies, the measurement was repeated at least five times with the same laser and fiber parameters. Afterwards, the mean values and the corresponding standard deviation were calculated and depicted in the graphs in the following sections.

\subsection{Influence of the pulse energy on the fiber degradation}

The effect of the pulse energy influence on the fiber degradation is shown in figure 4 (left). As expected, a high pulse energy leads to an increased fiber degradation. The deviation within the measurements with same pulse energies is probably caused by differences in the fiber. Small differences in the fiber material e.g. number and size of contaminations as well as possible changes in the refractive index and density can enhance or prevent the formation of a laser-induced damage. It is important to note that especially pulse energies below $350 \mu \mathrm{J}$ are of great interest. The reason for this is shown in figure 4 (right). It can be observed that pulse energies above $500 \mu \mathrm{J}$ always induce damages inside the fiber material thus leading to increased losses, and decreasing the transmitted pulse energy, after at least 40,000 pulses. Pulse energies at $350 \mu \mathrm{J}$ or below, not always lead to a reduction of the transmitted pulse energy. When transmitting low pulse energies of around $80 \mu \mathrm{J}$ and below, no degradation of the fiber material is expected.
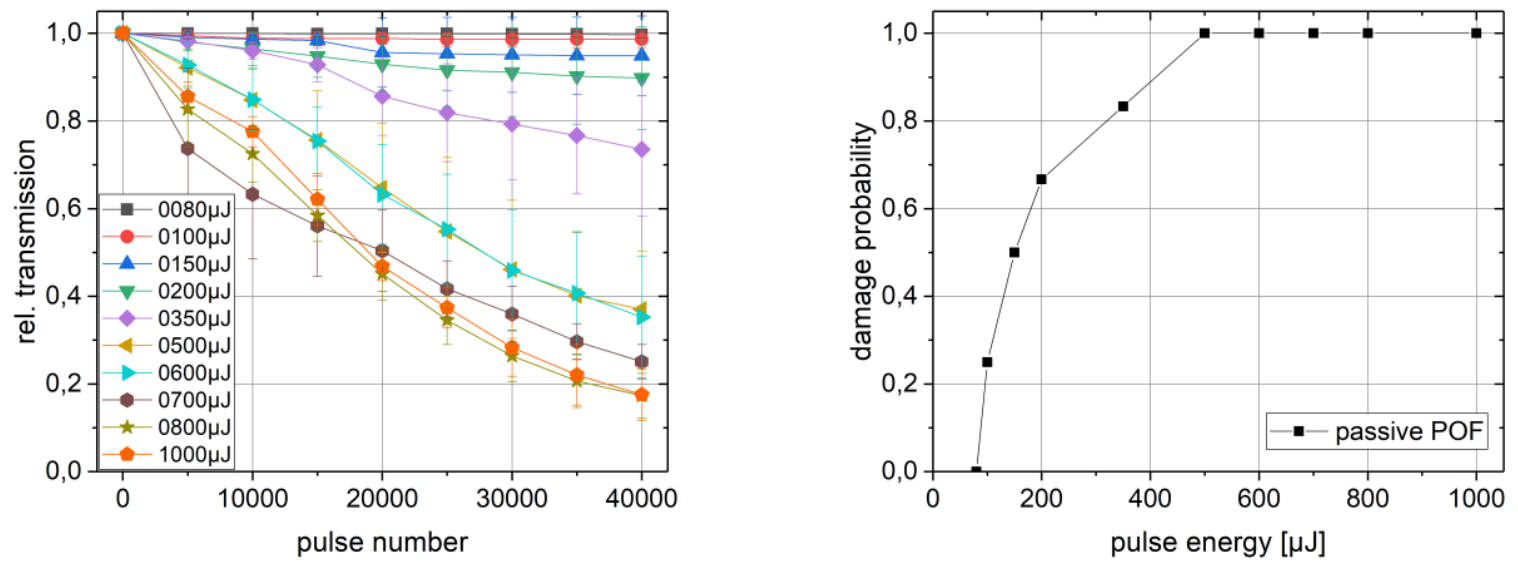

Figure 4. Influence of different pulse energies on the degradation of polymer optical fibers (left). Damage probability as a function of the pulse energy (right).

The trend of the relative transmission shows a saturation of the degradation for high pulse numbers. For better estimation of the saturation level, one fiber for each given pulse energy was exposed to at least 216,000 pulses and the output energy of the fibers was measured. It was observed that after the degradation is saturated, the pulse energy is always below $200 \mu \mathrm{J}$. This behavior probably correlates with the $0 \%$ damage probability. In the investigated series, the saturated output energy varied between 60 and $200 \mu \mathrm{J}$. This gap is most likely caused by smaller differences in the fiber core material already mentioned above leading to smaller changes in the damage generation. A variation in the degradation behavior is also expected, depending on the number of induced damages, the positions and the damage growth.

\subsection{Influence of the polymer chain length on the fiber degradation}

The relative transmission of two different fibers with a number-average molecular weight $\mathbf{M}_{n}$ of approximately 40,000 and 120,000 was monitored for different pulse energies (see figure 5 (left)).

It is observed that for 200 and $350 \mu \mathrm{J}$ only small deviations are notable between fibers with different chain lengths irradiated with the same pulse energy. Measurements at $500 \mu \mathrm{J}$ showed a comparable high difference in the transmission loss. However, the reference for the pulse energies are the same shown in figure 4 (left). In the latter, a conspicuous high 
degradation at a pulse energy of $500 \mu \mathrm{J}$ was already observed compared to different pulse energies. Overall, no significant influence of the polymer chain length on the laser-induced degradation was noticeable at 200 and $350 \mu \mathrm{J}$.
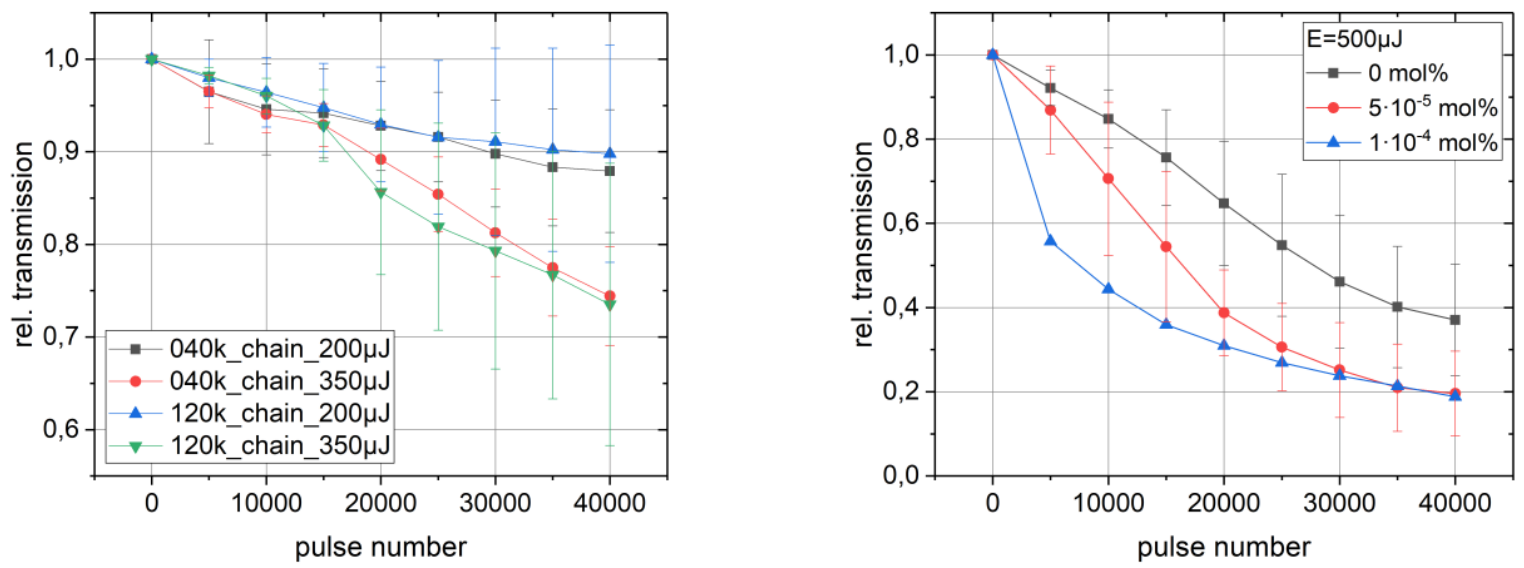

Figure 5. Influence of different polymer chain length (left) and the impact of different Rhodamine B concentrations (right) on the degradation of polymer optical fibers.

\subsection{Influence of the Rhodamine B concentration in active POFs on the fiber degradation}

In this part of the study, active polymer optical fibers were used to investigate the impact of different Rhodamine B concentrations on the fiber degradation at a pulse energy of $500 \mu \mathrm{J}$. The differences in the doping concentrations and core-/fiberdiameters compared to passive fibers used in this section are depicted in table 2. The corresponding measurement results are shown in figure 5 (right).

Table 2. Parameters of the fibers used in the investigation on the Rhodamine B concentration.

\begin{tabular}{|c|c|c|}
\hline Fiber & $\begin{array}{c}\text { Rhodamine B } \\
\text { concentration [mol\%] }\end{array}$ & $\begin{array}{c}\text { Core-/Fiberdiameter } \\
{[\boldsymbol{\mu m}]}\end{array}$ \\
\hline Passive POF & 0 & $450 / 520$ \\
\hline RB doped 1 & $5 \cdot 10^{-5}$ & $420 / 460$ \\
\hline RB doped 2 & $1 \cdot 10^{-4}$ & $430 / 470$ \\
\hline
\end{tabular}

As expected, the degradation increases with higher doping concentration. The Rhodamine B most likely acts as high absorber inside the core material favoring the damage process due to higher temperature. Moreover, Rhodamine B itself will bleach during the irradiation time influencing the relative transmission signal.

\section{MORPHOLOGY AND DISTRIBUTION OF LASER-INDUCED DAMAGES}

\subsection{Damage morphology}

In our previous study [4] we used Scanning Laser Optical Tomography [6] to reconstruct a highly detailed threedimensional image of some damaged sites for a first insight into the damage morphology. However, to collect sufficient data for a statistical analysis of the different morphologies, as well as the axial and radial damage distribution, a faster method using Nomarski microscopy was applied. To prevent refraction on the fiber interface, the fiber was embedded in glycerin and placed between two cover slips. Performing this index matching procedure, it was possible to study the laser-induced damages inside the fiber. Figure 6 shows one damage inside the fiber core at different angles. In figure 7 
three more damages can be seen. It is shown that the damage morphology varies, however a lenticular form is predominant in most cases, and the damage longitudinal axis is aligned perpindicular to the fibers longitudinal axis.
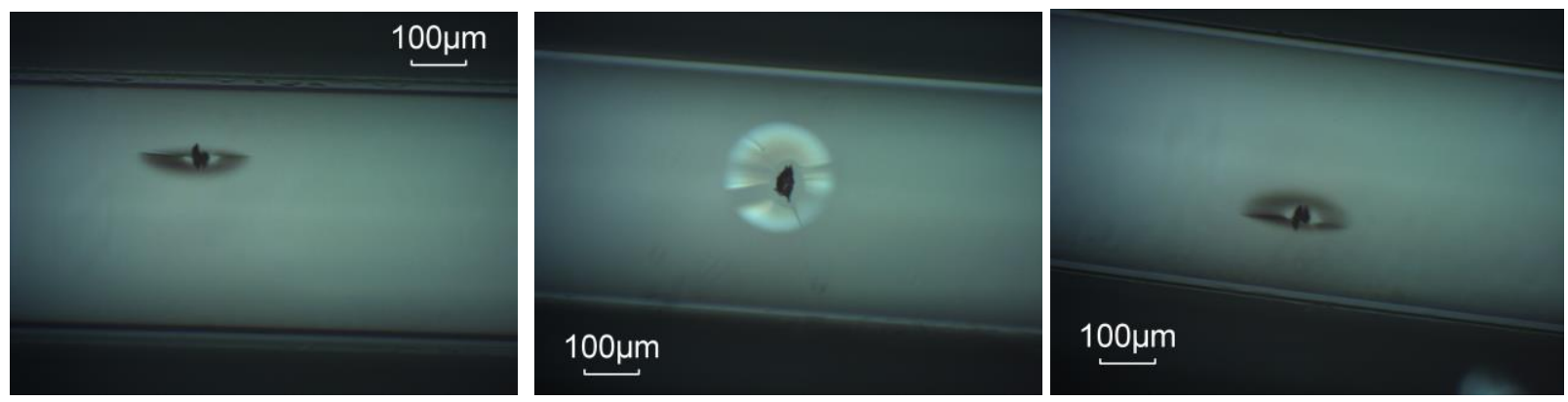

Figure 6. A laser-induced damage inside a polymer optical fiber at different angles captured using a Nomarski microscope.
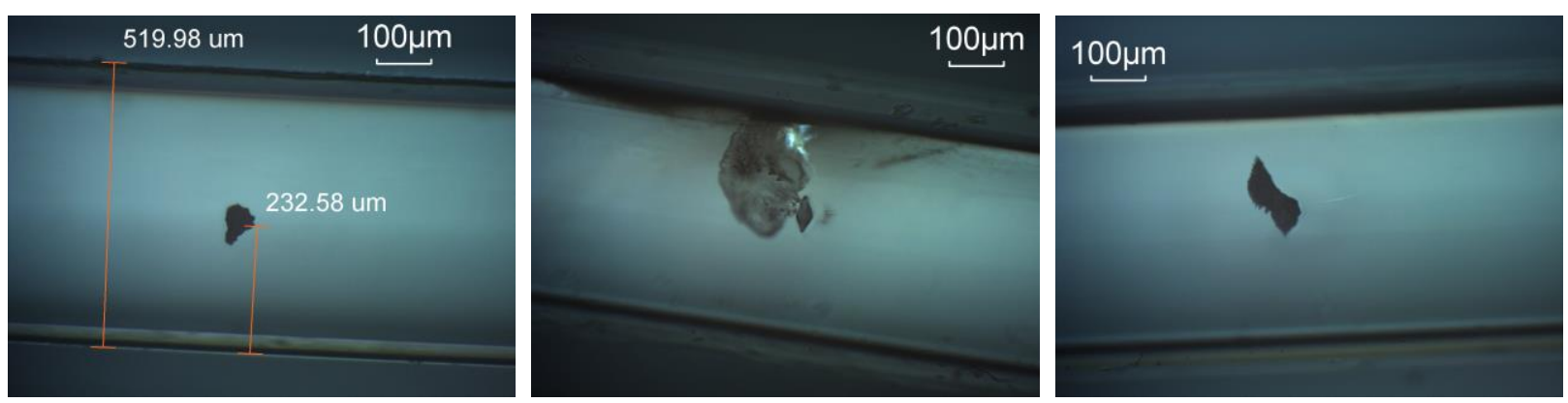

Figure 7. Three laser-induced damages in different POFs representing typical damage morphologies.

\subsection{Damage distribution}

Using Nomarski microscopy, the damage position along the fiber (figure 8 left) was determined using a standard scale. To determine the radial position (figure 8 right) of the damage inside the fiber, a rotation of the sample around its longitudinal axis can be performed during the microscopy process. In addition, a rough overview of the damage morphology at different viewing angles was possible. The measurement uncertainty of the axial position is estimated to be around $\pm 0.2 \mathrm{~mm}$ whereas the measurement uncertainty in the radial position amounts to roughly $\pm 25 \mu \mathrm{m}$.

The relative frequency of damages along the optical axis shows a rather homogenous distribution with a slight increase to the end. Overall a number of 22 fibers were studied regarding the damage position along the fiber. Therefore, the deviation of the homogenous behavior might be caused by the low number of fibers. However, during propagation inside the fiber, the intensity distribution changes. It is possible that this effect influences the damage formation. For this reason, further investigations on the damage cause and the intensity distribution are necessary to make a reliable statement. Regarding the damage density in dependence on the radial position, a decrease with increasing distance to the fiber core center is noticeable. Overall, considering the beam propagation inside an optical fiber and the beam profile shown in figure 2 (right), a higher beam intensity is expected in the center of the fiber core. Therefore, a higher damage density close to the core center is expected. 

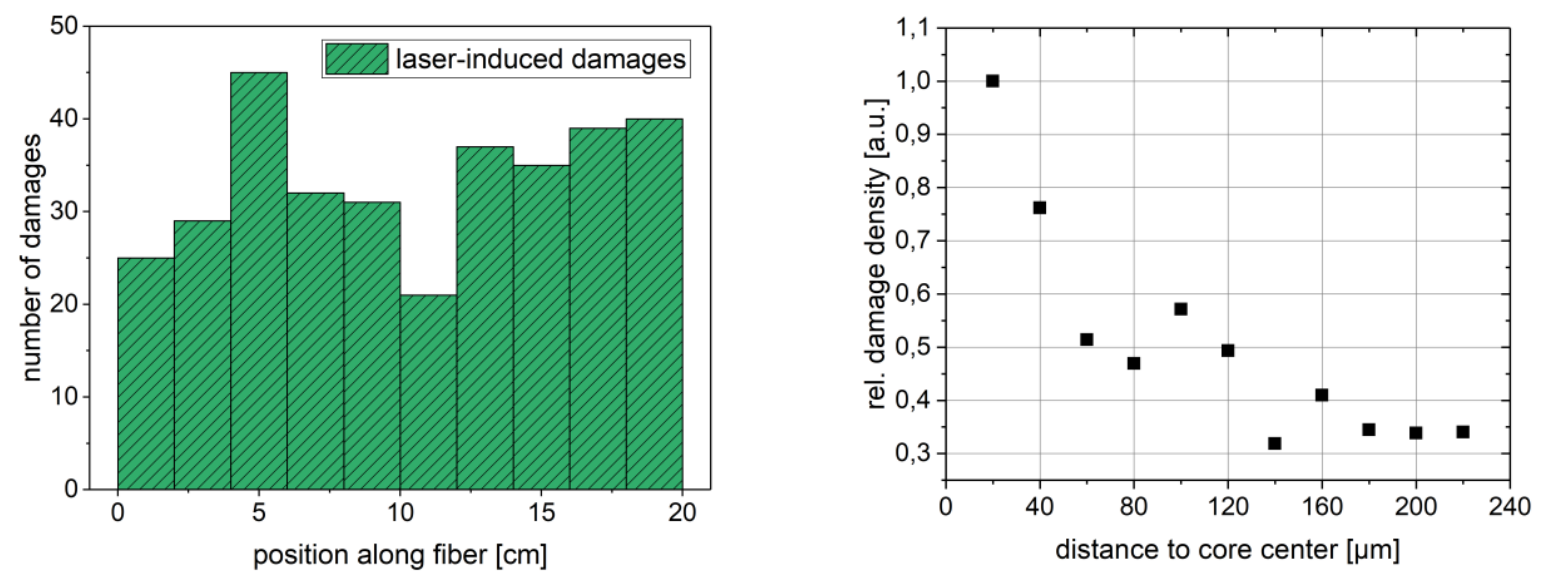

Figure 8. Relative number of laser induced damages along the POF (left). Damage density as a function of the distance to the core center (right).

\subsection{Damage formation}

First investigations indicate that the occurred damages inside the fiber core are cavities scattering the light which then partially exceeds the maximum angle of total reflection decreasing the transmission. It is assumed that these cavities grow during laser irradiation and reduce the transmission of the fiber. Finally, the scatter losses lead to a guided fluence below the laser-induced damage threshold inside the fiber, and the the degradation process is ceased. The nearly homogenous distribution of damages along the fiber as well as the survival curve and damage morphology indicate that the phenomenon is defect-induced. Moreover, similarities between the damages inside the preform material and the fiber core material lead to the assumption that the damaging process is most likely equivalent. Many investigations on the laser-induced damage mechanism of polymer material were already performed in the past by various researchers proposing different approaches extensively discussed in [7]. The transferability of these suggestions on the damage mechanism of optical fibers under nanosecond pulsed laser irradiation will be investigated in future studies. So far, in perspective to the damage mechanism mentioned in [7] it might be possible that high absorbing spots lead to locally high temperatures inducing pyrolysis of the fiber core material. This process forms carbon clusters accompanied by gaseous products leading to high pressure damaging the polymer. For example, the described procedure might have happened in figure 7 (center). However, further investigations on the damage process are necessary.

\section{CONCLUSION}

Different parameters were varied to investigate their influence on the laser-induced degradation in polymer optical fibers applying S-on-1 tests using nanosecond pulsed radiation at a wavelength of $532 \mathrm{~nm}$ of a Nd:YAG laser system. The degradation shows an "S"-shaped behavior saturating at an output energy below $200 \mu \mathrm{J}$ which corresponds to pulse energies below $100 \%$ damage probability. As expected, an intensification of the degradation behavior is observed with increasing pulse energy and increasing doping concentration. No significant impact on the degradation could be observed investigating different polymer chain lengths. In the present situation it is assumed that the damaging process is defectinduced, and future studies will focus on the underlying damage mechanisms. Therefore, further S-on-1 tests have to be performed regarding the impact of higher temperatures on the degradation, and it is necessary to further analyse the laser-induced damages in the irradiated material. 


\section{ACKNOWLEDGMENTS}

The authors thank the European Regional Development Fund for the financial support of the joint project LAPOF (contract no. ZW 685003502).

\section{REFERENCES}

[1] Steen, W. and Mazumder, J., [Laser Material Processing], Springer-Verlag, London (2010).

[2] Gebauer, J., Jupé, M., Bataviciute, G., Ristau, D. and Kling, R., "Measurement of laser power resistance of fiber for PIV systems," Proc. SPIE 7132 (2008).

[3] Wandschneider, R., Klein, K.-F. and Hillrichs G., "Transmission properties of polymer optical fibers for pulsed UV laser light," Proc. SPIE 6852 (2008).

[4] Kiedrowski, K., Thiem, J., Jakobs, F., Kielhorn, J., Balasa, I., Kracht, D. and Ristau, D., "Determiation of the laser-induced damage threshold of polymer optical fibers," Proc. SPIE 10805 (2018).

[5] Melninkaitis, A., Mikŝys, D., Maciulevicius, M. and Sirutkaitis, V., „Laser-induced damage threshold of stretched PMMA waveplates, “ Proc. SPIE 6403 (2006).

[6] Lorbeer, R., Heidrich, M., Lorbeer, C., Ojeda, D., Bicker, G., Meyer, H. and Heisterkamp, A., "Highly efficient 3D fluorescence microscopy with a scanning laser optical tomograph," Optics Express 19(6), 5419-5430 (2011)

[7] Fouassier, J.-P. and Rabek, J.F., [Lasers in Polymer Science and Technology: Applications], CRC Press, Boca Raton, Florida (1990). 